\title{
High frequency of pertussis in older children and adolescents with prolonged cough in Turkey
}

\author{
Aslı Aslan ${ }^{1}$, Zafer Kurugöl ${ }^{1}$, Şöhret Aydemir², Derya Gürsel ${ }^{2}$, Güldane Koturoğlu ${ }^{1}$ \\ Department of ${ }^{1}$ Pediatrics and ${ }^{2}$ Clinical Microbiology, Ege University Faculty of Medicine, Izmir, Turkey. \\ E-mail: zafer.kurugol@ege.edu.tr \\ Received: 18 January 2016, Revised: 15 March 2016, Accepted: 28 March 2016
}

SUMMARY: Aslan A, Kurugöl Z, Aydemir Ş, Gürsel D, Koturoğlu G. High frequency of pertussis in older children and adolescents with prolonged cough in Turkey. Turk J Pediatr 2016; 58: 41-46.

This study aimed to determine the frequency of B. pertussis infection among Turkish children with prolonged cough.

Nasopharyngeal specimens were collected from 7-18 year old children, presenting with prolonged cough of two to four weeks' duration. Specimens were examined for $B$. pertussis by PCR.

Of 101 children with prolonged cough, $20(19.8 \%)$ had a positive PCR testing for $B$. pertussis. Children who were vaccinated $\geq 5$ years previously had a 6.13-fold higher risk of PCR-confirmed pertussis than those who were vaccinated $<5$ years before. The classic symptoms of pertussis (paroxysmal cough, whooping and post-tussive vomiting) were seen in $30 \%, 15 \%$ and $25 \%$ of the patients with positive PCR, respectively; $55 \%$ of them had only a prolonged cough without any classic symptoms. Pertussis is common among Turkish children with prolonged cough, even after implementation of a fifth dose of pertussis vaccination and despite high vaccination coverage.

Key words: pertussis, prolonged cough, adolescents, real-time polymerase chain reaction, Turkey.

Pertussis is a highly contagious respiratory disease caused by the bacterium Bordetella pertussis. Among 194 World Health Organization (WHO) member states, 131 (68\%) countries had achieved $\geq 90 \%$ of three doses of diphtheria, tetanus, and pertussis vaccine (DTP3) coverage in 2012 and an estimated $83 \%$ of infants worldwide had received at least 3 doses of DTP vaccine ${ }^{1}$. However, despite the high vaccination coverage, pertussis continues to cause significant mortality and morbidity in many countries. WHO estimates that 16 million new cases of pertussis occur every year worldwide, with more than 195,000 deaths ${ }^{2}$.

WHO recommended that a pertussis incidence of $<1$ case per 100,000 population be achieved in Europe by $2000^{3}$. According to the data of the Turkish Ministry of Health, the incidence of pertussis decreased from 2.03/100,000 in 1986 to $0.04 / 100,000$ in 2013 . The number of pertussis cases has been reported as 48,242 , 18 and 33 for 2010, 2011, 2012 and 2013 respectively ${ }^{4}$. These data indicate that the goal of WHO has been achieved in Turkey. However, in our country, surveillance of pertussis relies on the clinical diagnosis notifications of practitioners and the actual incidence is probably much higher than reported cases.

Many different case definitions, such as those by the WHO, the US Centers for Disease Control and Prevention (CDC), European Union (EU) and Pan American Health Organization (PAHO) are currently used throughout the world ${ }^{5-7}$. However, there are difficulties in defining pertussis from a clinical perspective. In older children, adolescents and adults, the clinical course may not be typical, and persistent coughing may be the only symptom ${ }^{8,9}$. Even when symptoms are typical, pertussis may not be diagnosed because of the misconception among many physicians that pertussis is a childhood disease ${ }^{10}$. In these cases, the diagnosis of pertussis requires laboratory methods for confirmation. Laboratory confirmation tests 
include culture of $B$. pertussis, polymerase chain reaction (PCR) and serology. Most definitions (ie, WHO, CDC, EU) require culture and PCR assays that are specific for $B$. pertussis. However, there is no consensus internationally on the role of serology in the diagnosis of pertussis. The WHO definition requires paired serology, but the current $\mathrm{CDC}$ laboratory case definition does not include serology. The CDC recommends the use of both culture and PCR testing to confirm diagnosis ${ }^{6}$. This study was performed to determine the frequency of pertussis among Turkish children and adolescents with prolonged cough using real-time PCR technique.

\section{Material and Methods}

This study was conducted in the Department of Pediatrics of Ege University from August 2013 to August 2014. Children aged 7 to 18 years who presented with a prolonged cough of two to four weeks' duration were enrolled in the study. The protocol for the study was approved by the Ethical Committee and an informed consent was obtained from the parents or guardians of all eligible children.
Patients with an underlying medical condition which might cause a prolonged cough such as pulmonary tuberculosis, asthma, chronic bronchitis, chronic heart disease, sinusitis or who were immunocompromised, receiving longterm steroid therapy or angiotensin converting enzyme inhibitors or other medications known to cause cough were excluded.

Demographic variables, any related diseases or medications, duration of cough and the characteristic clinical manifestations of pertussis (whooping, paroxysmal cough and post-tussive vomiting) were recorded on a standard case report form for all patients. Immunization status for pertussis was obtained from the vaccination records.

Nasopharyngeal sampling (NFS) was taken easily from each patient by entering through nostrils and rotating the swabs that have flexible, twisted wire shafts with Dacron bud (Transwab, England). All the samples were sent to the bacteriology laboratory in the Department of Clinical Microbiology. Quantitative multiplex real-time PCR technique was used on these samples to amplification targets IS481 of B. pertussis. Commercial kit

Table I. Characteristic of Children With Positive and Negative PCR Test for B. Pertussis

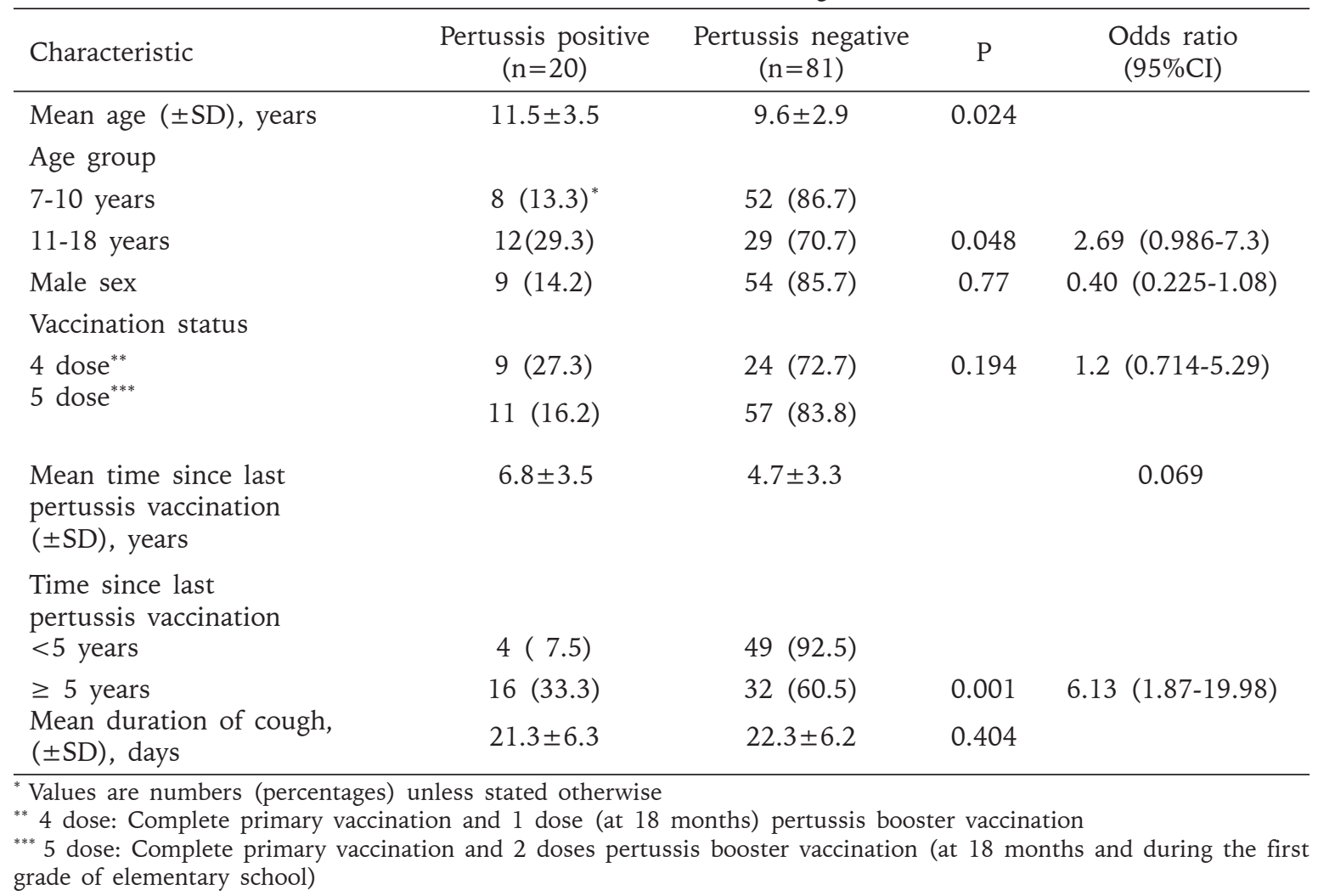


(LightMix kits, TIB Molbiol, GmbH, Germany) was used and all the analyses were done by LightCycler 1.5 (Roche Diagnostics, USA).

Baseline characteristics of study children were summarized by using numbers and percentages for categorical variables and means and $95 \%$ confidence intervals for continuous variables. Risk ratios were calculated with $95 \%$ confidence intervals in relation to a laboratory diagnosis of pertussis for age, sex, and vaccination status. We also calculated risk ratios with $95 \%$ confidence intervals for time since receiving the last pertussis vaccination. Vaccination status (four doses versus five doses of pertussis vaccine) and time since receiving the last pertussis vaccination $(<5$ years vs $\geq$ 5 years) was analyzed as a categorical variable. Statistical analysis was performed by SPSS 21.0 version for Windows.

\section{Results}

A total of 101 children who had a cough for 2-4 weeks were enrolled in the study; 63 (62.3\%) were male. The median age was 10 years (range, 7-18 years). There were 60 (59.4\%) children in the 7-10 year age group and $41(39.6 \%)$ children in the 11-18 year age group. The median duration of cough was 21 days (range, 14-28 days). All study children had received complete primary pertussis vaccinations (three doses of whole cell pertussis vaccine) and additional booster dose at 18 months; of whom $68(67.3 \%)$ had also received a fifth dose of pertussis vaccine (acellular pertussis vaccine containing two components) during the first grade of elementary school.

Out of 101 children with prolonged cough, $20(19.8 \%)$ had a positive PCR test for $B$. pertussis. Table 1 summarizes the characteristics of pertussis PCR positive and negative children. The mean age of the PCR positive patients was greater than those of PCR negative $(11.5 \pm 3.5$ vs $9.6 \pm 2.9$ years, $p=0.024)$. The rate of children with positive PCR increased significantly with age. About $13.3 \%$ of $7-10$ year old children and $29.3 \%$ of $11-18$ year old children had positive PCR $(p=0.048)$.

The risk of PCR confirmed pertussis was similar between children who had received four and five doses of pertussis vaccine (odds ratio 1.2, 95\% CI 0.714-5.29). However, increasing time since receiving last pertussis vaccination was associated with increased risk of pertussis. $B$. pertussis PCR was positive in $7.5 \%$ of children who had received the last vaccine less than 5 years previously. This rate went up to $33.3 \%$ with children who had received the last vaccination more than 5 years previously $(p=0.001)$. The risk of PCR confirmed pertussis was more than 6.13 times higher in children who had received the last pertussis vaccine five or more years previously than in patients who had received the last vaccine less than 5 years previously (odds ratio 6.13, 95\% CI 1.87-19.98).

Of the 101 children with prolonged cough, $33(32.7 \%)$ experienced a paroxysmal cough, $17(16.8 \%)$ had whooping and $36(35.6 \%)$ had post-tussive vomiting. There were no significant differences in the frequency of paroxysmal cough, whooping and post-tussive vomiting between children with positive and negative PCR (Table II). Of the 20 children with positive PCR for B. pertussis, only $6(30 \%)$ had paroxysmal cough, 3 (15\%) had whooping, and $5(25 \%)$ had post-tussive vomiting. While $9(45 \%)$ children with positive PCR had at least one symptom of pertussis, 11 (55\%) children had only a prolonged cough without any of characteristic features of pertussis (ie, paroxysmal cough, inspiratory whoop, and post-tussive vomiting).

\section{Discussion}

In the present study, $19.8 \%$ of children with prolonged cough had positive PCR for B. pertussis. Previous studies also reported from Turkey that pertussis was the cause in approximately $5-17 \%$ of cases with prolonged cough, despite of the fact that there was a range for the proportion of prolonged cough caused by $B$. pertussis because of the different study designs and various laboratory definitions ${ }^{11-13}$. The results show that pertussis is common among Turkish children and adolescents with prolonged cough, even after incorporation of a fifth dose of pertussis vaccine into the national immunization program and despite high levels of vaccination coverage. Despite this, physicians rarely diagnose and notify pertussis infection in these age groups.

Neither natural infection nor vaccination confers life-long protection against pertussis. Vaccineinduced immunity wanes, following whole-cell or acellular vaccines ${ }^{14}$. Studies demonstrate an increasing likelihood of pertussis after 
Table II. Symptoms of Children With Positive and Negative PCR test for B. pertussis

\begin{tabular}{lcccc}
\hline Symptoms & $\begin{array}{c}\text { Pertussis } \\
\text { positive } \\
(\mathrm{n}=20)\end{array}$ & $\begin{array}{c}\text { Pertussis } \\
\text { negative } \\
(\mathrm{n}=81)\end{array}$ & $\mathrm{P}$ & $\begin{array}{c}\text { Odds ratio } \\
(95 \% \mathrm{CI})\end{array}$ \\
\hline Paroxysmal cough & $6(30)^{*}$ & $27(33.3)$ & 0.53 & $0.91(0.29-2.83)$ \\
Whooping & $3(15)$ & $14(17.2)$ & 0.71 & $0.91(0.32-2.55)$ \\
$\begin{array}{l}\text { Post-tussive vomiting } \\
\begin{array}{l}\text { At least one symptom of } \\
\text { pertussis** }\end{array}\end{array}$ & $5(25)$ & $31(38.2)$ & 0.79 & $1.05(0.39-2.97)$ \\
\hline
\end{tabular}

*Values are numbers (percentages) unless stated otherwise

** Children with cough for 2-4 weeks and at least one symptom of paroxysmal cough, whooping and post-tussive vomiting

completion of a five dose series of acellular pertussis vaccinations (DTaP) given at the ages of $2,4,6$, and 15-18 months and 4-6 years. In a large cohort analysis of fully vaccinated children, risk ratios for pertussis after the fifth dose of DTaP increased from 1.9 (95\% confidence interval 1.3 to 2.9$)$ in year 2 to 8.9 (6.0 to 13.0 ) in year $6^{15}$. The other study, from the United States, reported that protection from pertussis after a fifth dose of DTaP among children who had received only DTaP vaccines was relatively short-lived and waned substantially each year and risk of pertussis increase by an average of $42 \%$ a year during the first five years ${ }^{16}$. A recent cohort study, from United Kingdom, suggested that the risk of pertussis was more than threefold higher in children who had received the preschool pertussis booster vaccination seven years or more previously, $59 \%$ of whom received three doses of whole cell pertussis vaccine ${ }^{17}$. Our findings similarly show that the risk of pertussis was more than six times higher for children who had received the last pertussis vaccination five or more years earlier, even though all children had received complete primary pertussis vaccinations with whole cell pertussis vaccine and $68 \%$ of them had also received a fifth dose of pertussis vaccine during the first grade of elementary school.

Several types of laboratory tests are used in the laboratory diagnosis of $B$. pertussis infection. Common laboratory diagnostic tests currently include culture, PCR and serologic study on acute and convalescent-phase sera. Sensitivity and specificity of the tests depends on the age of the patient, time from symptom onset, vaccination and exposure history, specimen collection and test procedure ${ }^{18}$.
Culture is considered the gold standard laboratory test and is specific for $B$. pertussis but its sensitivity is very low. Fastidious growth requirements make $B$. pertussis difficult to culture ${ }^{19}$. Culture may provide accurate results during the first two weeks of illness when viable bacteria are still present in the nasopharynx. After the first two weeks of cough, the sensitivity of culture declines and the risk of false-negatives increase. Furthermore, culture has limited sensitivity for previously vaccinated persons, especially adolescents and adults.

Several commercial serologic test kits with a variety of pertussis antigens and varying degrees of sensitivity and specificity are available. However, diagnosing pertussis using commercial serological kits is problematic ${ }^{18}$. The serologic tests are not well standardized; the accuracy of test result varies between different kits, and the variety of antigens used ${ }^{20}$. Serological diagnosis is based on a significant increase in antibody titer or on a single high antibody titer above an age-adjusted cutoff. However, cutoff points for diagnostic values of IgG antibody to pertussis toxin (PT) have not been established, and current IgA and IgM assays lack adequate sensitivity and specificity. Recent vaccination can influence the positivity of the antibody identification; immune response to vaccination cannot be distinguished from response to infection ${ }^{5}$. Anti-PT IgG and IgA antibodies are detectable in most vaccinated children, adolescents, and adults. There is also no consensus internationally on the role of serology in the diagnosis of pertussis. WHO accepts seroconversion or a significant increase in antibody level but not a single high titer. In the US, these are not recommended 
for routine use in establishing a diagnosis of pertussis because CDC believes that "no serologic method for diagnosis of pertussis has been validated between laboratories or has been accepted for diagnostic use in US" 21 . Serologic tests are generally useful for diagnosis in later phases of the disease. Serology may be performed on specimens collected at least 2 weeks following cough onset, but the optimal timing of specimen collection for single-serum serology is 4 to 8 weeks post-cough onset ${ }^{22}$.

Polymerase chain reaction testing is an important tool for timely diagnosis of pertussis and is widely used for diagnosis of pertussis since $2001^{23}$. PCR is a molecular technique used to detect DNA sequences of the B. pertussis bacterium, and unlike culture, does not require viable bacteria present in the specimen ${ }^{24}$. Compared with culture, PCR is more sensitive than culture for diagnosis of pertussis and remains positive until significantly later in disease than culture and is recommended for diagnosis in adolescents and adults ${ }^{25-29}$. PCR is a rapid test and has excellent sensitivity ${ }^{18}$. For PCR, the optimal timing for specimen collection is the first 3 weeks of cough, but bordetella DNA may be detected by PCR for 4 weeks after cough onset ${ }^{18,23}$. After the fourth week of illness, the amount of bacterial DNA rapidly diminishes, which increases the risk of obtaining falsely-negative results. In vaccinated children, adolescents and adults with cough illness of $\leq 3$ weeks' duration, PCR is the only appropriate method for diagnosis of pertussis $18,30,31$. A PCR method with high sensitivity such as IS481 is ideal for the evaluation of sporadic coughing illness in the age groups who are almost always either previously immunized against or exposed to B. pertussis ${ }^{30}$. In the present study, IS481 PCR assay was performed for diagnosis of pertussis in vaccinated children and adolescents with a cough of two to four weeks' duration. However, disadvantages to PCR include its relatively high cost, more limited availability, and the potential for false-negative or false-positive results ${ }^{32,33}$.

In this study, $30 \%$ of our PCR-confirmed pertussis cases had paroxysmal cough, $15 \%$ had whooping, and $25 \%$ had post-tussive vomiting. A previous study of adolescents and adults with persist cough from Massachusetts US, found paroxysms in $83 \%$ of cases, whooping in $30 \%$ and vomiting in $45 \%$. Paroxysm was the most common symptom ${ }^{34}$. In another study, from Iran, detected pertussis by culture and PCR in school-age children with persistent cough, the frequencies of these symptoms were $59.1 \%, 55.5 \%$ and $22.3 \%$, respectively; $85 \%$ of the children had at least one symptom of paroxysmal cough, whooping, or post-tussive emesis $^{35}$. In our study, $55 \%$ of PCR-confirmed cases had only a prolonged cough without any of classic findings of pertussis. Our results suggest that prolonged cough may be the only symptom of pertussis infection in older children and adolescents ${ }^{36}$.

The present study shows that pertussis can still cause clinically significant prolonged cough in Turkish children and adolescents who have received complete primary pertussis vaccinations and the fifth dose booster vaccination. However, physicians' awareness of the infection is very poor. Pertussis is rarely considered in differential diagnosis of prolonged cough in older children and adolescents, and remains an underrecognized and underdiagnosed condition. Adolescents are significant reservoirs for the organism and are frequent sources of infection for infants and unvaccinated children. Therefore, vaccination of adolescents will protect both themselves and unvaccinated or not yet fully immunized infants.

\section{REFERENCES}

1. Global routine vaccination coverage-2012. Centers for Disease Control and Prevention (CDC.) MMWR Morb Mortal Wkly Rep, 2013; 62: 858-861.

2. Pertussis vaccines: WHO position paper. Wkly Epidemiol Rec 2010; 85: 385-400.

3. Centralized Information System for Infectious Diseases (CISID). Pertussis incidence. Available from: URL: http://data.euro.who.int/cisid/?TabID=366495

4. Cherry JD, Tan T, Wirsing von König CH, et al. Clinical definitions of pertussis: Summary of a Global Pertussis Initiative roundtable meeting, February 2011. Clin Infect Dis 2012; 54: 1756-1764.

5. Centers for Disease Control and Prevention (CDC). Pertussis / Whooping Cough (Bordetella pertussis) 2014 Case Definition. Available from: URL: http:// wwwn.cdc.gov/nndss/conditions/pertussis/casedefinition/2014.

6. World Health Organization: WHO. Recommended Standards for Surveillance of Selected Vaccine Preventable Disease, Vaccine Assessment and Monitoring team of the Department of Vaccines and Biologicals; 2003: 28.

7. Couzigou C, Flahault A. Is pertussis being considered as a cause of persistent cough among adults? Eur J Epidemiol 2003; 18: 1013-1015. 
8. Cherry JD. Pertussis vaccines for adolescents and adults. Pediatrics 2005; 116: 755-756.

9. Cherry JD, Grimprel E, Guiso N, Heininger U, Mertsola J. Defining pertussis epidemiology: clinical, microbiologic and serologic perspectives. Pediatr Infect Dis J 2005; 24: S25-34.

10. Dilli D, Bostanci I, Dallar Y, Buzgan T, Irmak $\mathrm{H}$, Torunoğlu MA. Recent findings on pertussis epidemiology in Turkey. Eur J Clin Microbiol Infect Dis 2008; 27: 335-341.

11. Yildirim I, Ceyhan M, Kalayci O, et al. Frequency of pertussis in children with prolongued cough. Scand J Infect Dis 2008 ;40: 314-319.

12. Aksakal FN, Cöplü N, Ceyhan MN, et al. High incidence of Pertussis among schoolchildren with prolonged cough in Turkey. Tohoku J Exp Med 2007; 211: 353358 .

13. Mink CA, Sirota NM, Nugent S. Outbreak of pertussis in a fully immunized adolescent and adult population. Arch Pediatr Adolesc Med 1994; 148: 153-157.

14. Tartof SY, Lewis M, Kenyon C, White K, Osborn A, Liko J, et al. Waning immunity to pertussis following 5 doses of DTaP. Pediatrics 2013; 131: 1047-1052.

15. Klein NP, Bartlett J, Rowhani-Rahbar A, Fireman B, Baxter R. Waning protection after fifth dose of acellular pertussis vaccine in children. N Engl J Med 2012; 367: 1012-1019.

16. Wang K, Fry NK, Campbell H, et al. Whooping cough in school age children presenting with persistent cough in UK primary care after introduction of the preschool pertussis booster vaccination: prospective cohort study. BMJ 2014; 348: 3668.

17. Riffelmann $\mathrm{M}$, Wirsing von Konig $\mathrm{CH}$, Caro $\mathrm{V}$, et al. Nucleic acid amplification tests for diagnosis of Bordetella infections. J Clin Microbiol 2005; 43: 49254929.

18. Kennerknecht N, Riffelmann M, Schmetz J, Wirsing von König $\mathrm{CH}$. Comparison of commercially available immunoblot assays measuring IgG and IgA antibodies to Bordetella pertussis antigens. Eur J Clin Microbiol Infect Dis 2011; 30: 1531-1535.

19. Murphy T, Bisgard K, Sanden G. Diagnosis and laboratory methods, chapter 2. Guidelines for the control of pertussis outbreaks, National Immunization Program. Centers for Disease Control and Prevention, (CDC). 2000

20. Menzies SL, Kadwad V, Pawloski LC, et al. Pertussis Assay Working Group. Development and analytical validation of an immunoassay for quantifying serum anti-pertussis toxin antibodies resulting from Bordetella pertussis infection. Clin Vaccine Immunol 2009; 16: 1781-1788.

21. Kline JM, Lewis WD, Smith EA, Tracy LR, Moerschel SK. Pertussis: a reemerging infection. Am Fam Physician 2013; 88: 507-514.

22. Palmer CM, McCall B, Jarvinen K, Nissen MD. Bordetella pertussis PCR positivity, following onset of illness in children under 5 years of age. Commun Dis Intell Q Rep 2007; 31: 202-205.
23. Koidl C, Bozic M, Burmeister A, Hess M, Marth E, Kessler HH. Detection and differentiation of Bordetella spp. by real-time PCR. J Clin Microbiol 2007; 45: 347-350.

24. Schmidt-Schläpfer G, Liese JG, Porter F, Stojanov S, Just $\mathrm{M}$, Belohradsky $\mathrm{BH}$. Polymerase chain reaction (PCR) compared with conventional identification in culture for detection of Bordetella pertussis in 7153 children. Clin Microbiol Infect 1997; 3: 462-467.

25. Lind-Brandberg L, Welinder-Olsson C, Lagergård T, Taranger J, Trollfors B, Zackrisson G. Evaluation of PCR for diagnosis of Bordetella pertussis and Bordetella parapertussis infections. J Clin Microbiol 1998; 36: 679-683.

26. Heininger U, Schmidt-Schlapfer G, Cherry J, Stehr $\mathrm{K}$. Clinical validation of polymerase chain reaction assay for the diagnosis of pertussis by comparison with serology, culture, and symptoms during a large pertussis vaccine efficacy trial. Pediatrics 2000; 105 : 31.

27. Bidet P, Liguori S, De Lauzanne A, et al. Real-time PCR measurement of persistence of Bordetella pertussis DNA in nasopharyngeal secretions during antibiotic treatment of young children with pertussis. J Clin Microbiol 2008; 46: 3636-3638.

28. Bonacorsi S, Farnoux C, Bidet P, Caro V, Aizenfisz S, Benhayoun $\mathrm{M}$ et al. Treatment failure of nosocomial pertussis infection in a very-low-birth-weight neonate. J Clin Microbiol 2006; 44: 3830-3832.

29. McIntyre PB, Sintchenko V. The "how" of polymerase chain reaction testing for Bordetella pertussis depends on the "why". Clin Infect Dis 2013; 56: 332-334.

30. Guiso N, Berbers G, Fry NK et al. What to do and what not to do in serological diagnosis of pertussis: recommendations from EU reference laboratories. Eur J Clin Microbiol Infect Dis 2011; 30: 307-312.

31. Dragsted DM, Dohn B, Madsen J, Jensen JS. Comparison of culture and PCR for detection of Bordetella pertussis and Bordetella parapertussis under routine laboratory conditions. J Med Microbiol 2004 ;53: 749-754.

32. Mandal S, Tatti KM, Woods-Stout D, et al. Pertussis pseudo-outbreak linked to specimens contaminated by Bordetella pertussis DNA from clinic surfaces. Pediatrics 2012; 129: 424-430.

33. Yih WK, Lett SM, des Vignes FN, et al. The increasing incidence of pertussis in Massachusetts adolescents and adults, 1989-1998. J Infect Dis 2000; 182: 1409-1416.

34. Ghanaie RM, Karimi A, Sadeghi H, et al. Frequency of pertussis in iranian school-age children. J Ped Infect Dis 2013; 8: 1-5.

35. Ghanaie RM, Karimi A, Sadeghi H, et al. Sensitivity and specificity of the World Health Organization pertussis clinical case definition. Int J Infect Dis 2010; 14: 1072-1075.

36. Bremont F, Micheau P, Le RP, Brouard J, Pin I, Fayon $\mathrm{M}$. Etiology of chronic cough in children: analysis of 100 cases. Arch Pediatr 2001; 8: 64-69. 